\title{
Pretreatment Parameters Obtained from Peripheral Blood Sample Predicts Invasiveness of Bladder Carcinoma
}

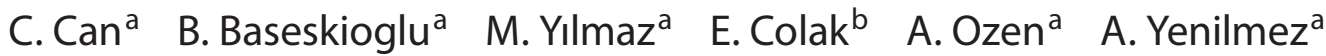 \\ Departments of a Urology and ${ }^{b}$ Biostatistics, Osmangazi University Faculty of Medicine, Eskişehir, Turkey
}

\section{Key Words}

Urothelial carcinoma $\cdot$ Neutrophil $\cdot$ Lymphocyte $\cdot$

Neutrophil-to-lymphocyte ratio $\cdot$ Platelets $\cdot$ Logistic

regression model

\begin{abstract}
Purpose: To predict the invasiveness of urothelial bladder carcinoma using a logistic regression model on preoperative peripheral blood samples. Patients and Methods: Hospital data of patients operated for urothelial carcinoma were reviewed retrospectively. Preoperative blood samples were collected before the first cystoscopic examination. Any kind of infection or inflammation was an exclusion criterion. Patients were grouped as having a non-muscle-invasive or muscle-invasive urothelial carcinoma. The mean age was 69 years and was determined as the cut-off value. According to receiver operating characteristic curves, threshold points were determined for lymphocytes, neutrophil count, neutrophil-to-lymphocyte ratio (NLR), thrombocytes and mean platelet volume. Demographic specialties, parameters obtained from blood samples, tumor size and multiplicity were evaluated and significant parameters were put into a logistic regression model. Results: The study group consisted of 80 non-muscle-invasive and 102 muscle-invasive patients. Age ( $\leq 69$ vs. $>69$ ), female gender, NLR (2.57), mean platelet vol-
\end{abstract}

ume $(7.9 / \mathrm{fl})$ and platelet count $(400,000 / \mu \mathrm{l})$ were significant parameters and put in a model. Using odds ratios, the probability of tumor invasiveness was calculated by a formula. Conclusion: Age, female gender, NLR and platelet count were found to be the predictors of invasiveness of urothelial carcinoma.

Copyright $\odot 2012$ S. Karger AG, Basel

\section{Introduction}

Bladder cancer is a common tumor in the urinary tract and is the ninth most prevalent cancer around the world [1]. Approximately $90 \%$ of bladder cancers are urothelial carcinomas (UC) and $75-85 \%$ of UC are non-muscleinvasive while 15-25\% are invasive [2]. Treatment of UC depends on pathological behavior of the tumor. Nonmuscle-invasive tumors can be removed by transurethral resection but recurrence is common. Intracavitary BCG therapy is one of the prophylaxis and treatment options for non-muscle-invasive UC. Many investigations suggest that one of the anti-tumor mechanisms of BCG is related to local immunological responses, since BCG induced local infiltration of the bladder wall with activated T cells, macrophages, natural killer (NK) cells and B cells [3] and MHC class II antigen on urothelial cells [4]. BCG

\section{KARGER}

Fax +4161306 1234

E-Mail karger@karger.ch

www.karger.com (c) 2012 S. Karger AG, Basel

0042-1138/12/0894-0468\$38.00/0

Accessible online at:

www.karger.com/uin
Barbaros Baseskioglu, MD

Osmangazi University Faculty of Medicine

Department of Urology

Meselik, Eskișehir (Turkey)

E-Mail barbaroza@gmail.com 
instillation involved not only local immunological efforts but also systemic immune responses [5]. In other words, UC and the immune system are supposed to be associated with each other.

Changes in host inflammatory responses and tumor relations have been increasingly recognized in various tumor types, and inflammatory cells around the tumor cells play a significant role in the progress and prognosis of tumors. Mostly neutrophils with relative lymphocytopenia or thrombocytosis are the indicators of systemic immune response-tumor interactions. Tissue neutrophils may be associated with a poor prognosis [6]. Also, an increased neutrophil-to-lymphocyte ratio (NLR) has been shown as a poor prognostic factor in patients with malignancies [7].

The association of thrombocytosis with cancer was described initially by Levin and Conley [8] in 1964, with the finding that $38 \%$ of adult patients with thrombocytosis had an unrecognized primary cancer. One hypothesis is that growing tumors induce thrombocytosis by secretion of growth factors and cytokines. IL-6, which is one of them, is able to stimulate platelet production. Thrombocytosis (platelet count $>400 \times 10^{9} / \mathrm{l}$ ) has been reported to be a poor prognostic factor in many cancers because of its association with an increased expression of angiogenic factors [9].

To summarize, immunity and UC seem to be involved with each other. This study attempts to explain this relation with a logistic regression model prepared from peripheral blood samples for predicting the invasiveness of UC.

\section{Patients and Methods}

\section{Patients}

For this retrospective study, we reviewed the surgical and pathological reports of 267 patients with UC operated between 2001 and 2011 under the permission of the ethical committee of the hospital. Among this population, 85 cases were excluded because of referral patients with pathological diagnoses from other centers and unavailable data. Also, patients with other histological types of bladder cancer, acute inflammatory diseases, any evidence of infection, myeloproliferative disorders, autoimmune diseases, splenectomies and patients using drugs which may affect platelet count and function were excluded from the study. Thus, the study population consisted of 182 patients. Patients were examined physically, and urinalyses and urine cultures were taken. All patients had undergone cystoscopic evaluation. Specimens were obtained by transurethral resection. Pathological examination of the specimens were carried out by two senior pathologists.

Predictors of Invasiveness of Urothelial Carcinoma
Blood Analysis

Samples for full blood count analysis were collected preoperatively into ethylenediaminetetraacetic acid (EDTA) anticoagulated tubes. Measurements were performed on a Coulter Counter model Max M (Coulter Diagnostics, Hialeah, Fla., USA). Mean platelet volume (MPV) assay was standardized according to the manufacturer's instructions. The Coulter Counter model Max M uses the S-CAL_ kit to calibrate the white blood cell count, red blood cell count, hemoglobin level, mean corpuscular volume, platelet count, and MPV. Calibration was monitored twice daily. In order to avoid the platelet swelling induced by EDTA, blood samples were analyzed within half an hour.

\section{Statistics}

Factors analyzed were patient age, gender, lifestyle, occupational environment, tumor stage, platelet count, tumor size $(\leq 3$ vs. $>3 \mathrm{~cm}$ ), tumor multiplicity, MPV, neutrophil count, lymphocyte count and NLR. Continuous variables were changed into categorical variables according to approximate optimal cut-off points which were analyzed by receiver operating characteristic curves (ROCs). The mean value of age ( $\leq 69 \mathrm{vs.}>69$ ) was used for analysis. The relations between pathological stage and parameters were analyzed by logistic regression analysis and odds ratios were calculated. All p values $<0.05$ were considered statistically significant. All statistical analysis was performed with the Statistical Package for Social Sciences (SPSS) version 17.0. In a logistic regression model, a 95\% CI including 1 was accepted as insignificant.

\section{Results}

The study population was divided into two groups, i.e. muscle-invasive urothelial tumors $(n=102)$ and non-muscle-invasive urothelial tumors $(\mathrm{n}=80)$. The demographics for the two groups are summarized in table 1. Preoperatively, none of the patients had any signs of systemic or local infection, including urinary tract infection. The most common mode of presentation was hematuria and all patients had a history of smoking. The mean follow-up was 45 months with a range of $6-180$ months.

According to ROCs, the optimal threshold values of NLR, neutrophil, lymphocyte, platelet and MPV counts were determined as $2.57,6,500 / \mu \mathrm{l}, 1,600 / \mu \mathrm{l}, 400,000 / \mu \mathrm{l}$ and 7.9/fl, respectively. Tumor size, tumor multiplicity, occupational environment and lifestyle were of no help in predicting tumor invasiveness $(\mathrm{p}>0.05)$. A logistic regression model was built with these parameters and correlated with pathological stage as non-muscle-invasive or invasive. All parameters had predictive values and odds ratios (table 2). NLR had the most significant $p$ value in correlation with pathological stage while the platelet count had the most risk. When we sorted out the hazard 
ratios of these parameters from strongest to weakest, it could be summarized as platelet count $>$ female gender $>$ NLR $>$ age.

\section{Preoperative Pathological Stage Predictability Model}

Using the parameters in the logistic regression model, the risk of primary tumor invasiveness can be calculated by formula 1 . The logistic regression model for the probability of having an invasive UC preoperatively can be demonstrated as:

$$
\pi\left(x_{1}, x_{2}, x_{3}, x_{4}, x_{5}\right)=\frac{e^{\beta_{1} x_{1}+\beta_{2} x_{2}+\beta_{3} x_{3}+\beta_{4} x_{4}+\beta_{5} x_{5}}}{1+e^{\beta_{1} x_{1}+\beta_{2} x_{2}+\beta_{3} x_{3}+\beta_{4} x_{4}+\beta_{5} x_{5}}}
$$

where

$$
\begin{aligned}
& x_{1}=\left\{\begin{array}{ll}
1 & \text { NLR }>2.57 \\
0 & \text { NLR } \leq 2.57
\end{array}, \quad x_{2}= \begin{cases}1 & \text { Platelet }>400 \mu \mathrm{l} \\
0 & \text { Platelet } \leq 400 \mu \mathrm{l}\end{cases} \right. \\
& x_{3}= \begin{cases}1 & \text { Mean platelet volume }>7.9 \mathrm{fl} \\
0 & \text { Mean platelet volume } \leq 7.9 \mathrm{fl}\end{cases} \\
& x_{4}=\left\{\begin{array}{ll}
1 & \text { Age }>69 \\
0 & \text { Age } \leq 69
\end{array}, \text { and } x_{5}= \begin{cases}1 & \text { Female Gender } \\
0 & \text { Male Gender }\end{cases} \right.
\end{aligned}
$$

The $\beta s$ are the coefficients of the variables $\left(x_{1}, \ldots, x_{5}\right)$ and obtained from the logistic regression analysis as $\beta_{1}=$ $1.022, \beta_{2}=1.438, \beta_{3}=0.664, \beta_{4}=0.804$, and $\beta_{5}=1.359$. The corresponding formula can be obtained as:

$$
\pi\left(x_{1}, x_{2}, x_{3}, x_{4}, x_{5}\right)=\frac{e^{1.022 x_{1}+1.438 x_{2}+0.664 x_{3}+0.804 x_{4}+1.359 x_{5}}}{1+e^{1.022 x_{1}+1.438 x_{2}+0.664 x_{3}+0.804 x_{4}+1.359 x_{5}}}
$$

Putting each variable into this formula gives the relative risk for having an invasive UC preoperatively.

\section{Discussion}

UC comprises more than $90 \%$ of all bladder cancers, while adenocarcinoma and squamous cell carcinoma are much less common [10]. UC have been classified into two groups with distinct behaviors: non-muscle-invasive and muscle-invasive. Clinically, non-muscle-invasive UC accounts for $75-85 \%$ of neoplasms, while $15-25 \%$ of UC are invasive to muscle or beyond [2]. Bladder cancer is more common in men than women, as shown in this study [11]. This situation can be explained by the higher rates of smokers in men. Also, males have higher 5-year survival rates than women and the factors which cause reduced mortality are uncertain [12]. Survival by stage at presen-
Table 1. Demographic distribution of patients

\begin{tabular}{lll}
\hline Parameters & $\begin{array}{l}\text { Non-muscle-invasive } \\
(\mathrm{n}=80)\end{array}$ & $\begin{array}{l}\text { Muscle-invasive } \\
(\mathrm{n}=102)\end{array}$ \\
\hline $\begin{array}{l}\text { Sex } \\
\text { Male }\end{array}$ & $76(95 \%)$ & $86(84.3 \%)$ \\
$\quad$ Female & $4(5 \%)$ & $16(15.7 \%)$ \\
Age & & \\
$\quad \leq 69$ years & $60(75 \%)$ & $55(53.9 \%)$ \\
$>69$ years & $20(25 \%)$ & $47(46.1 \%)$ \\
Size & & $15(14.7 \%)$ \\
$\quad \leq 3$ cm & $37(46.3 \%)$ & $85(85.3 \%)$ \\
$\quad>3$ cm & $43(53.8 \%)$ & $13(12.7 \%)$ \\
Tumor number & & $89(87.3 \%)$ \\
$\quad$ Solitary & $19(23.8 \%)$ & \\
$\quad \begin{array}{l}\text { Multiple } \\
\text { Appearance }\end{array}$ & $61(76.3 \%)$ & $16(45.1 \%)$ \\
$\quad$ Solid & & $39(38.2 \%)$ \\
$\quad \begin{array}{l}\text { Papillary } \\
\text { Mixed }\end{array}$ & $2(2.5 \%)$ & \\
\hline
\end{tabular}

Table 2. Logistic regression model for significant parameters

\begin{tabular}{llll}
\hline Variables & OR & $95 \%$ CI & $\mathrm{p}$ \\
\hline NLR $>2.57$ & 2.7800 & $1.3830-5.5883$ & 0.004 \\
Platelet $>400 \mu \mathrm{l}$ & 4.2160 & $1.1274-15.7663$ & 0.032 \\
Mean platelet volume $>7.9 \mathrm{fl}$ & 1.9430 & $0.9848-3.8333$ & 0.055 \\
Age $>69$ years & 2.2359 & $1.1051-4.5241$ & 0.025 \\
Female gender & 3.8953 & $1.0741-14.1269$ & 0.038 \\
\hline
\end{tabular}

tation is one of the most favorable factors and this is confirmed in this study as females have 3.8 times more invasive cancer.

Bladder cancer can occur at any age but the incidence is increased in the elderly with a median of 69 years in males and 71 years in females. In patients younger than 40 years, bladder cancer tends to be well differentiated. Inversely, in elderly people the bladder cancer is more aggressive [13]. Advanced stage at diagnosis, impaired host defenses or less-aggressive therapies are probable factors. Similarly, age above 69 years is 2.2 times more likely to be an invasive tumor in this study.

High-grade or muscle-invasive tumors are more likely to metastasize. The standard treatment for UC is transurethral resection of tumors, after which about $60-70 \%$ of tumors will recur, and $25 \%$ will progress to a higher grade or stage [14]. The ability of tumor invasion and metastasis is dependent both on the intrinsic characteristics 
of the tumor cells and on the environment around the tumor [15]. The abnormal phenotype of the tumor may stimulate an influx of inflammatory lymphocytes into tissues surrounding the tumor [16]. The systemic inflammatory response also features changes in the relative levels of circulating white blood cells [17]. There is increasing evidence that cell-mediated immunity plays a key role in the tumor immunology of patients with cancer. A systemic inflammatory response is assessed by the concentration of acute-phase proteins, e.g. CRP, fibrinogen, ferritin, albumin and transferrin, or peripheral blood leukocyte components, including neutrophils and lymphocytes [7]. Increasing evidence supports the involvement of systemic inflammation in cancer development and progression [18]. It has been reported for various types of cancer that the degree of systemic inflammation reflects the local tumor burden. O’Toole et al. [19] reported a correlation between lymphocyte reactivity against bladder tumor cells and clinical stage. In that study, increased lymphocyte counts were shown in $88 \%$ of the patients with non-muscle-invasive bladder carcinoma but in only $41 \%$ of those with infiltrating tumor. Predicting the prognosis of patients with cancer by examination of peripheral blood leukocytes would seem to be an easier and useful procedure [20]. A report has shown that healthy individuals with a high NLR ratio have a significantly lower NK activity than those with a low NLR ratio [21]. Impaired cellular and humoral immune responses have been described in various malignant diseases. Hirashima et al. [20] showed a correlation between the NLR ratio of the patients with early gastric cancer and the prognosis of the patients. Kishi et al. [22] showed that NLR independently predicts survival in patients with colorectal liver metastases treated with chemotherapy followed by resection or chemotherapy only. When chemotherapy normalizes the high NLR, improved survival is expected. Also, in one study, elevated preoperative NLR was identified as an adverse predictor of outcome in patients undergoing potentially curative resection for hepatocellular carcinoma, and, therefore, patients with high preoperative NLR should be considered candidates for additional therapies after resection [23]. The combination of $\mathrm{T}$ stage and the NLR ratio can be used to stratify the risk of recurrence in patients with nonmetastatic renal cell carcinoma [17]. Increased preoperative NLR is associated with poor survival in cases of colorectal carcinoma [16]. Also, these results indicate that a simultaneous increase of neutrophils and decrease of lymphocytes is important for determination of the prognosis [20]. Well-recognized neutrophilia is accompanied by a relative lymphocytopenia. The NLR

Predictors of Invasiveness of Urothelial Carcinoma has been suggested as a simple index of systemic inflammatory response in critically ill patients [24]. In our study, we found a correlation between pathological stage and age, NLR, female gender and platelet count with logistic regression analysis. These parameters can be used to predict the pathological stage of the tumor before the treatment. NLR is an easily measurable parameter of systemic inflammation, which can predict pathological stage.

Thrombocytosis may adversely affect survival by facilitating cell invasion and metastasis. A number of studies have suggested that platelets play an integral role in the metastatic process. There is evidence that platelets protect tumor cells by shielding them from the host's immune system [25]. Platelets also facilitate tumor cell adhesion to the vascular endothelium by forming tumor thrombi and through interactions between the tumor and platelet ligands. $\mathrm{P}$ selectin on activated platelets has an important role in tumor growth and metastasis. Vascular endothelial growth factor, a recognized critical component of tumor angiogenesis, thrombocytosis may be an end result of growth factors secreted by tumor cells and the host response or it may be an event that directly increases the risk of spreading the disease and worsens the prognosis [26]. Thrombocytosis has repeatedly been identified as a marker of advanced RCC with an independent prognostic value [27]. The platelet count was found to significantly correlate with tumor size, tumor stage and surgical margin status but was not found to correlate with a histological subtype of renal cell carcinoma. The platelet count (both categorically $>400 \times 10^{9}$ cells/l and as a continuous variable) was identified to significantly predict decreased overall and disease-specific survival. In another study, thrombocytosis was more common among patients with more advanced stage disease in gastric cancer [28]. According to Todenhöfer et al. [29], the presence of thrombocytosis before radical cystectomy is a poor prognostic factor. They also commented that preoperative thrombocytosis may be used as an additional parameter to identify bladder cancer patients with advanced disease.

\section{Conclusion}

The pathological stage of the tumor is the most important factor in the treatment of UC. Early treatments such as intravesical agents or cystectomy are strongly recommended according to pathological stage. As known, the time period between transurethral resection and cystectomy effects survival. Also, early intravesical treatments 
are shown to decrease tumor recurrence. Our invasiveness risk formula may be useful in the decision of early staging after transurethral resection in likely to be muscle-invasive tumors. Also, this study may be one of the signs of a relationship existing between peripheral blood and UC.

\section{Disclosure Statement}

The authors have no financial disclosures to make.

\section{References}

1 Ferlay J, Shin HR, Bray F, et al: Cancer Incidence and Mortality Worldwide: International Agency for Research on Cancer, 2010. Available from: http://globocan.iarc.fr.

$\checkmark 2$ Jaeger N, Radeke HW, Adolphs HD, et al: Value of intravesical sonography in tumor classification of bladder carcinoma. Eur Urol 1986;12:76-84.

3 Sakai I, Miyake H, Harada KI, et al: Analysis of factors predicting intravesical recurrence of non-muscle invasive transitional cell carcinoma of the bladder without concomitant carcinoma in situ. Int J Urol 2006;13:13891392.

4 Prescott S, James K, Hargreave TB, et al: Intravesical Evans strain BCG therapy: quantitative immunohistochemical analysis of the immune response within the bladder wall. J Urol 1992;147:1636-1642.

5 Taniguchi K, Koda S, Nishikido M, et al: Systemic immune response after intravesical instillation of bacille Calmette-Guerin (BCG) for non-muscle invasive bladder cancer. Clin Exp Immunol 1999;115:131-135.

6 Yamashita J, Ogawa M, Shirakusa T: Free form neutrophil elastase is an independent marker predicting recurrence in primary breast cancer. J Leukoc Biol 1995;57:375378.

7 Ohno Y, Nakashima J, Ohori M, et al: Pretreatment neutrophil-to-lymphocyte ratio as an independent predictor of recurrence in patients with nonmetastatic renal cell carcinoma. J Urol 2010;184:873-878.

8 Levin J, Conley CL: Thrombocytosis associated with malignant disease. Arch Intern Med 1964;114:497-500.

-9 Sierko E, Wojtukiewicz MZ: Platelets and angiogenesis in malignancy. Semin Thromb Hemost 2004;30:95-108.
10 Nascimento CM, Drager BJ, Zeegers MP, et al: Epidemiology of urinary bladder cancer: from tumor development to patient's death. World J Urol 2007;25:285-295.

11 Jemal A, Murray T, Ward E, et al: Cancer statistics, 2005. CA Cancer J Clin 2005;55:1030.

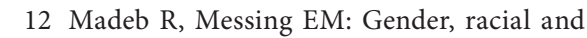
age differences in bladder cancer incidence and mortality. Urol Oncol 2004;22:86-92.

13 Linn JF, Sesterhenn I, Mostofi FK, et al: The molecular characteristics of bladder cancer in young patients. J Urol 1998;159:14931496.

14 Totterman TH, Loskog A, Essand M: The immunotherapy of prostate and bladder cancer. BJU Int 2005;96:728-735.

15 Yamada H, Luo Y, Matsumoto T, et al: A novel expression of macrophage derived chemokine in human bladder cancer. J Urol 2005; 173:990-995.

16 McMillan DC, Canna K, McArdle CS: Systemic inflammatory response predicts survival following curative resection of colorec tal cancer. Br J Surg 2003;90:215-219.

17 Walsh SR, Cook EJ, Goulder F, et al: Neutrophil-lymphocyte ratio as a prognostic factor in colorectal cancer. J Surg Oncol 2005;91: 181-184.

18 Mantovani A, Allavena P, Sica A, et al: Cancer related inflammation. Nature 2008; 454 : 436-444.

19 O’Toole C, Perlman P, Unsgaard B, et al: Cellular immunity to human urinary bladder carcinoma. Correlation to clinical stage and radiotherapy. Int J Cancer 1972;10:77-91.

20 Hirashima M, Higuchi S, Sakamoto K, et al: The ratio of neutrophils to lymphocytes and the phenotypes of neutrophils in patients with early gastric cancer. J Cancer Res Clin Oncol 1998;124:329-334.
21 Lasek WA, Plodziszewska M, Gorecki D, et al: Relationship between natural killer activity and eosinophil, neutrophil and lymphocyte level in human blood. Folia Haematol 1985;117:907-912.

22 Kishi Y, Kopetz S, Chu YS, et al: Blood neutrophil-to lymphocyte ratio predicts survival in patients with colorectal liver metastasis treated with systemic chemotherapy. Ann Surg Oncol 2009; 16:614-622.

23 Gomez D, Farid S, Malik HZ, et al: Preoperative neutrophil-to lymphocyte ratio as a prognostic predictor after curative resection for hepatocellular carcinoma. World J Surg 2008;32:1757-1762.

24 Zahorec R: Ratio of neutrophil-to-lymphocyte counts. Rapid and simple parameter of systemic inflammation and stress in critically ill. Bratisl Lek Listy 2001;102:5-14.

25 Nieswandt B, Hafner M, Echtenacher B, et al: Lysis of tumor cells by natural killer cells in mice is impeded by platelets. Cancer Res 1999;59:1295-1300.

26 Kim YJ, Borsig L, Varki NM, et al: P-selectin deficiency attenuates tumor growth and metastasis. Proc Natl Acad Sci USA 1998;95: 9325-9330.

27 Suppiah R, Shaheen PE, Elson P, et al: Thrombocytosis as a prognostic factor for survival in patients with metastatic renal cell carcinoma. Cancer 2006;107:1793-1800.

28 Ikeda M, Furukawa H, Imamura $\mathrm{H}$, et al: Poor prognosis associated with thrombocytosis in patients with gastric cancer. Ann Surg Oncol 2002;9:287-291.

29 Todenhöfer T, Renninger M, Schwentner C, et al: A new prognostic model for cancer-specific survival after radical cystectomy including pretreatment thrombocytosis and standard pathological risk factors. BJU Int DOI: $10.1111 / j .1464-410 X .2012 .11231 . x$. 